Original scientific paper

\title{
DEMAND PREDICTION AND ALLOCATION OPTIMIZATION OF MANUFACTURING RESOURCES
}

\author{
Meng, J. L. \\ School of Economics and Management, Harbin University, Harbin 150086, China \\ E-Mail: mengiinglei420@126.com
}

\begin{abstract}
Big data analysis, Internet technology, and cloud computing are being integrated to the allocation of production and manufacturing (P-M) resources, promoting the transformation, upgrading, and innovative development of traditional job-shop P-M resource allocation. However, the existing studies have not fully considered the demand dynamicity of production materials. To solve the problem, this paper attempts to predict the demand and optimize the allocation of job-shop P-M resources. Firstly, a demand prediction model was established for job-shop P-M resources, which can simultaneously capture the static and dynamic spatial dependence of P-M resource volume. Based on the demand prediction, the authors detailed an allocation optimization strategy for job-shop P-M resources, and defined the objective function and constraints. The proposed model was proved effective through experiments.

(Received in August 2021, accepted in November 2021. This paper was with the author 1 month for 1 revision.)
\end{abstract}

Key Words: Intelligent Manufacturing, Allocation Optimization, Demand Prediction, Production and Manufacturing (P-M) Resources

\section{INTRODUCTION}

With the growing intelligence and automation of production and manufacturing (P-M) methods, the competition across and within industries becomes increasingly fierce [1-5]. Against this backdrop, enterprises need to improve their ability to effectively reduce cost and respond to market demand changes [6-10]. Big data analysis, Internet technology, and cloud computing are being integrated to the allocation of production and manufacturing (P-M) resources, promoting the transformation and upgrading of traditional job-shop P-M resource allocation [11-17]. To innovate the allocation of P-M resources, some job-shop P-M resource supply management systems have emerged. These systems provide platforms that receives delivery demand and information, and thereby connect delivery participants.

Mashhadi and Monroy [18] constructed a multi-objective optimization model for resource allocation, which considers the interests of manufacturing resource demanders and cloud platform operators in the cloud environment, and solved the model by the dynamic neighbourhood algorithm. Zhang and $\mathrm{Yu}$ [19] proposed a unified production resource allocation method, which makes rapid decisions on allocating resources to the task of the inserted order, according to the resource processing information and machine evaluation strategy [20]. Delaram et al. [17] discussed how platform, matching algorithm, and resource availability influence the utility of manufacturing suppliers and consumers, and developed a framework to obtain managerial insights and to choose the most suitable matching algorithm for each scenario. Pei et al. [21] put forward a multi-level manufacturing service network, and provided a dynamic resource allocation strategy for each node based on the queuing theory; On this basis, a closed approximation algorithm and a numerical method were proposed to dynamically allocate the manufacturing resources of each node, corresponding to each target level of the manufacturing service network. Brintha et al. [22] quantified the matching between tasks and resources with an adaptive search mechanism, presented a heuristic method based on the bat algorithm, and thereby balanced computing time, makespan, and workload. Yang and Chen [23] discussed the 
application of fuzzy resource allocation algorithm in virtual job-shops, and explored the realtime job scheduling of job-shops.

Many models and algorithms have been developed for P-M resource allocation. However, most of the studies refer to the traditional logistics modes to examine operation mechanism and path optimization, yet fail to fully consider the dynamicity of production resource demand. This paper predicts the demand and optimize the allocation of job-shop P-M resources. The main contents are as follows: (1) Setting up a demand prediction model for job-shop P-M resources, which can simultaneously capture the static and dynamic spatial dependence of P-M resource volume; (2) Based on the demand prediction, an allocation optimization strategy was detailed for job-shop P-M resources, along with the objective function and constraints. The proposed model was proved effective through experiments.

\section{MODEL CONSTRUCTION}

Grid-based P-M resource prediction often combined traditional convolutional neural network (CNN) with long short-term memory network (LSTM). But the combinatory model overlooks the time dependence of the prediction problem. To solve the problem, this paper constructs a deep bidirectional spatiotemporal network, which employs the bidirectional LSTM (BiLSTM) (Fig. 1) to capture the time dependence of time series in different directions. Meanwhile, the proposed network can extract the P-M resource volumes at the same moments in a week, and extract the entire P-M resource volume series of several days. The extracted data enable the modelling under long-term cyclic demand, as well as under short-term dynamic cyclic demand.

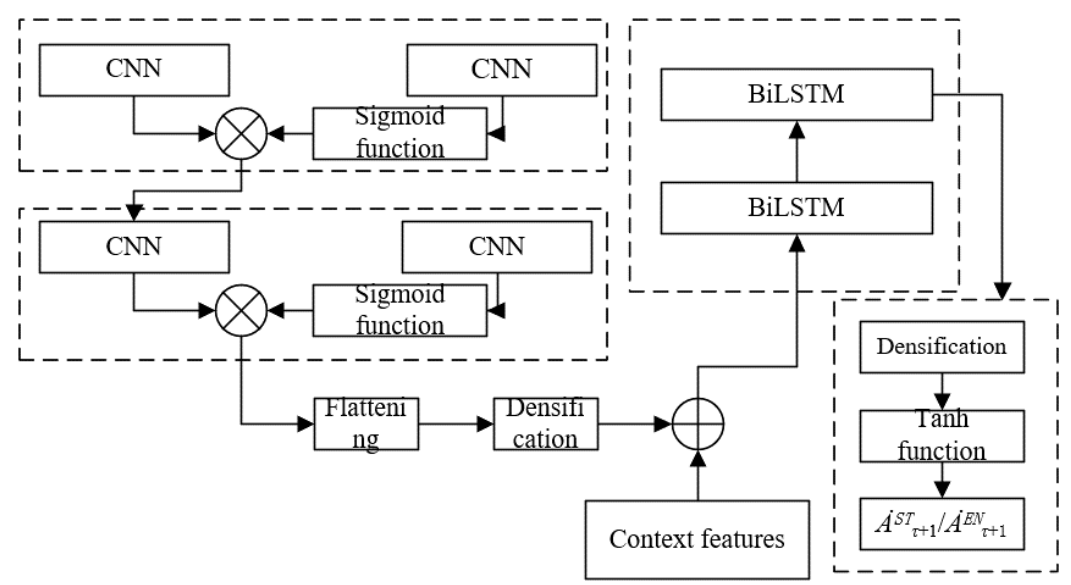

Figure 1: Structure of the prediction model.

The entire job-shop was divided into $n$ resource allocation zones (RAZs) of equal size $x \times y$. Then, the RAZ set of the job-shop can be described as: $K=\left\{k_{1}, \ldots, k_{d}, \ldots, k_{n}\right\}$. Then, the P-M resource delivery in each RAZ was recorded by a fixed interval, forming a set of delivery times: $D T=\left\{D T_{1}, \ldots, D T_{s}, \ldots, D T_{P}\right\}$, where $P$ is the total number of time periods. For simplicity, time period $D T_{s}$ and RAZ $k_{d}$ are denoted by $\tau$ and $d$, respectively. Let $A^{S T}{ }_{\tau, d}$ be the number of trajectories of the initial P-M resource volume departing from RAZ $d$ at time $\tau$, and $A^{E N}{ }_{\tau, d}$ be those of the final P-M resource volume arriving at $\mathrm{RAZ} d$ at time $\tau$. In the meantime, the inflow and outflow series of vehicles were established in the deep bidirectional spatiotemporal network. Let $g^{I N}{ }_{\tau, d}$ be the number of trajectories of vehicle inflow moving from another RAZ at time $\varepsilon$ $(\varepsilon \leq \tau)$ and arriving at RAZ $d$ at time $\tau ; g^{O U}{ }_{\tau, d}$ be the number of trajectories of vehicle outflow moving from RAZ $d$ at time $\varepsilon(\varepsilon \leq \tau)$ and arriving at another RAZ at time $\tau$.

By the above definitions, it is possible to determine the initial and final resource volume series, as well as the vehicle inflow and outflow series for each RAZ at time $\tau$. On this basis, the initial and final resource volumes could be predicted for each RAZ at time $\tau+1$. 


\subsection{Capturing nonlinear dependence}

At any time $\tau$, the proposed model constructs the P-M resource volumes of the target RAZ and its adjacent RAZs as a two-channel tensor $T C T_{\tau, d} \in R^{Q \times Q^{\times 2 E}}$, where $\mathrm{Q}$ is the spatial space being covered. $T C T_{\tau, d}$ contains two channels, namely, the initial P-M resource volume $T C T^{S T}{ }_{\tau, d}$ and the final P-M resource volume $T C T^{E N}{ }_{\tau, d}$.

In the deep bidirectional spatiotemporal network, the static spatial dependence is captured by importing the P-M resource volume tensor $T C T_{\tau, d}$ of the target RAZ into a D-layer local $\mathrm{CNN}$. Let $\omega_{\tau}^{l}$ and $y_{\tau}^{l}$ be learnable hyperparameters, which satisfy $g(c)=\max (c, 0)$. Then, the operation of the $l^{\text {th }}$ convolutional layer can be expressed as:

$$
\begin{gathered}
\operatorname{TCT}_{\tau, d}^{l}=g(c) \\
c=\omega_{\tau}^{l} * A_{\tau, d}^{l-1}+y_{\tau}^{l}
\end{gathered}
$$

To capture the dynamic spatial dependency of P-M resource data, our model analyses and depicts the dynamic relationship between P-M RAZs. For the target RAZ $d$, the P-M resource flow in the past $N$ time periods was stacked in the form of P-M resource flow tensor $G_{\tau, d} \in R^{Q \times Q^{\times 2 E}}$, and imported to the local CNN. Let $\omega_{\tau^{\prime}}^{l}$ and $y_{\tau^{\prime}}^{l}$ be the learnable parameters. Then, the operation of the $l^{\text {th }}$ layer can be expressed as:

$$
G_{\tau, d}^{l}=g\left(\omega_{\tau^{\prime}}^{l} * G_{\tau, d}+y_{\tau^{\prime}}^{l}\right)
$$

To control the propagation of the potential spatial information in the P-M resource flow, it is necessary to capture the static and dynamic spatial dependence of P-M resource volume synchronously. For this purpose, this paper constructs a flow gating mechanism. Let $\odot$ be the Hadamard product between corresponding elements; $B_{\tau, d}^{l}$ be the output of the $l^{\text {th }}$ layer; $B_{\tau, d}^{0}$ be the inputted P-M resource volume tensor $T C T_{\tau, d}$. After being processed by the flow gating mechanism, the operation of the $l^{\text {th }}$ layer can be expressed as:

$$
B_{\tau, d}^{l}=g\left(\omega_{\tau}^{l} * B_{\tau, d}^{l-1}+y_{\tau}^{l}\right) \odot \gamma\left(G_{\tau, d}^{l}\right)
$$

After the operation of the $L$-layer $C N N$, the proposed deep bidirectional spatiotemporal network will import the output $B^{K}{ }_{\tau, d}$ of the $L^{\text {th }}$ layer to the flattening layer and the fully connected layer, producing the expression $b_{\tau, d}$ of the high-level potential space.

The traditional LSTM, capable of effectively capturing the nonlinear correlation between the data series of P-M resource volume, can be introduced to obtain the time dependence for P$\mathrm{M}$ resource prediction. However, the traditional LSTM could not capture the time dependence of the inverse time series for P-M resources. Hence, the traditional LSTM was replaced by BiLSTM in our model. The BiLSTM could capture the time dependence of both positive and inverse time series for predicting P-M resources.

The BiLSTM contains two traditional LSTMs. That is, the P-M resource data of positive and inverse time series are separately placed in different LSTM layers to learn the time dependence in different directions. Let $\omega_{h}, \delta_{h}$, and $b_{h}(h \in\{h, f, \beta, \psi\})$ be all learnable parameters; $h_{\tau, d}, g_{\tau, d}$, and $E_{\tau, d}$ be the outputs of the input gate, forget gate, and output gate of LSTM, respectively; $\gamma$ be the sigmoid function, i.e., the activation function; $o_{\tau, d}$ be the contextual feature for modelling; $b_{\tau, d}$ be the potential space expression; $f_{\tau, d}$ be the output of the LSTM. Then, the calculation process of each LSTM can be expressed as:

$$
\begin{aligned}
& h_{\tau, d}=\gamma\left(\omega_{i}\left\lfloor b_{\tau, d} ; o_{\tau, d}\right\rfloor+\delta_{i} f_{\tau-1, d}+y_{i}\right) \\
& g_{\tau, d}=\gamma\left(\omega_{g}\left\lfloor b_{\tau, d} ; o_{\tau, d}\right\rfloor+\delta_{g} f_{\tau-1, d}+y_{g}\right) \\
& E_{\tau, d}=\gamma\left(\omega_{\beta}\left\lfloor b_{\tau, d} ; o_{\tau, d}\right\rfloor+\delta_{\beta} f_{\tau-1, d}+y_{o}\right)
\end{aligned}
$$




$$
\begin{gathered}
\psi_{\tau, d}=\tanh \left(\omega_{\psi}\left\lfloor b_{\tau, d} ; o_{\tau, d}\right\rfloor+\delta_{\psi} f_{\tau-1, d}+y_{\psi}\right) \\
u_{\tau, d}=g_{\tau, d} * u_{\tau-1, d}+u_{\tau-1, d}+h_{\tau, d} * \psi_{\tau, d} \\
f_{\tau, d}=\beta_{\tau, d} * \tanh \left(u_{\tau, d}\right)
\end{gathered}
$$

Let $f_{\tau}^{\prime}$ and $f^{\prime \prime}{ }_{\tau}$ be the outputs of the positive LSTM layer and the inverse LSTM layer, respectively. The BiLSTM combines the outputs of the two traditional LSTMs by the cascading function $g_{C B}$ to get the final output:

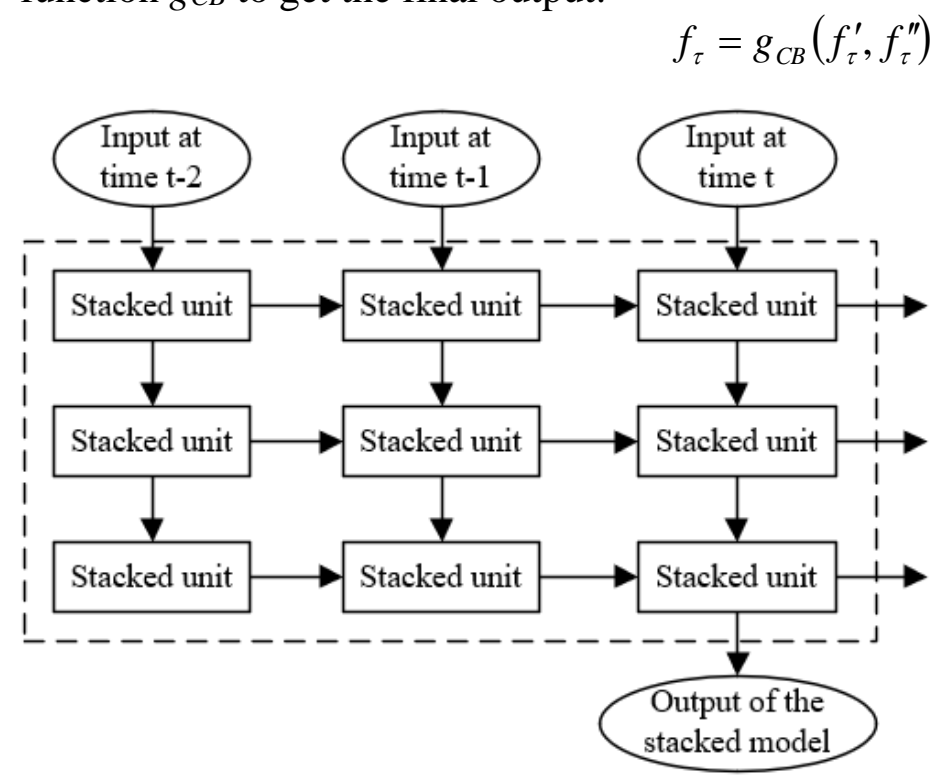

Figure 2: Architecture of deep bidirectional spatiotemporal network.

The multilayer architecture of the deep bidirectional spatiotemporal network (Fig. 2) was constructed through stacking. The output of each layer is inputted to the next layer of the bidirectional spatiotemporal network.

\subsection{Construction of prediction model}

Although the P-M resource data clearly change periodically, the law of periodic variation may go out of control in the short term, under the interference of external factors. If an excessively long P-M resource data series is imported to the model, vanishing gradients may occur to the BiLSTM, which undermines the learnability of long-term dependence. To avoid the above problem, our model establishes a short-term and a long-term variation sequences of P-M resource volume, based on the trajectories of vehicles in a fixed period. The long-term set of P$\mathrm{M}$ resource volumes was created based on the P-M resource volumes at the same moment in several weeks, and the short-term set of P-M resource volumes was created based on all P-M resource volumes during several days in the target RAZ.

Suppose one day contains a total of SF time periods. The long-term and short-term sets of P-M resource volumes of RAZ $d$ can be expressed as $C O_{d}=\left\{c O_{\tau-w \times S F, d}, \ldots, c O_{\tau-S F, d}\right\}$ and $D O_{d}=$ $\left\{D O_{\tau^{-} v \times S F, d}, D O_{\tau^{-} v \times S F+1, d}, \ldots, D O_{\tau-1, d}\right\}$, respectively; the lengths of the long-term and short-term sets of P-M resource volumes can be depicted as $w$ and $v$, respectively. After being processed by the bidirectional LSTM, the proposed deep bidirectional spatiotemporal network will import the output to the fully-connected layer, generating the predicted P-M resource volume. Let $\dot{A}^{S T}{ }_{\tau+1} / \dot{A}^{E N}{ }_{\tau+1}$ be the predicted initial and final P-M resource volumes at time $\tau+1$ of all RAZs, respectively; $\omega_{O U}$ and $y_{O U}$ be learnable parameters. Then, we have:

$$
\left\lfloor\dot{A}_{\tau+1}^{S T}, \dot{A}_{\tau+1}^{E N}\right\rfloor=\tanh \left(\omega_{O U} f_{\tau}^{L}+y_{O U}\right)
$$


Let $\lambda$ be a hyperparameter. To predict the effect of prediction error on model prediction, the following loss function can be defined:

$$
Q S=\lambda\left(\dot{A}_{\tau+1}^{S T}-A_{\tau+1}^{S T}\right)^{2}+(1-\lambda)\left(\dot{A}_{\tau+1}^{E N}-A_{\tau+1}^{E N}\right)^{2}
$$

\section{ALLOCATION OPTIMIZATION}

\subsection{Generation of prediction demand}

All signals of P-M resource delivery come from the supply management system of job-shop P$\mathrm{M}$ resources, which consists of three parts: system, demand points, and vehicles. The demand points send resource demand to the system, looking for suitable vehicles to transport the P-M resources. The system predicts the demand according to the historical demand of the demand points, and provides the following services in the light of the current information of resource delivery: vehicle allocation, route optimization, in-transit tracking, etc. The vehicles deliver the resources, and feedback real-time delivery information to the system. Fig. 3 shows the operation mode of the system. To predict the real-time request for job-shop P-M resources more accurately, the allocation range and delivery time of $\mathrm{P}-\mathrm{M}$ resources were reasonably arranged according to the spatiotemporal distribution of real-time resource demands issued to the delivery region $R$ of the supply management system of job-shop P-M resources. Each RAZ $C A$ and allocation period $C T$ constitute a prediction unit, $d \in R$.

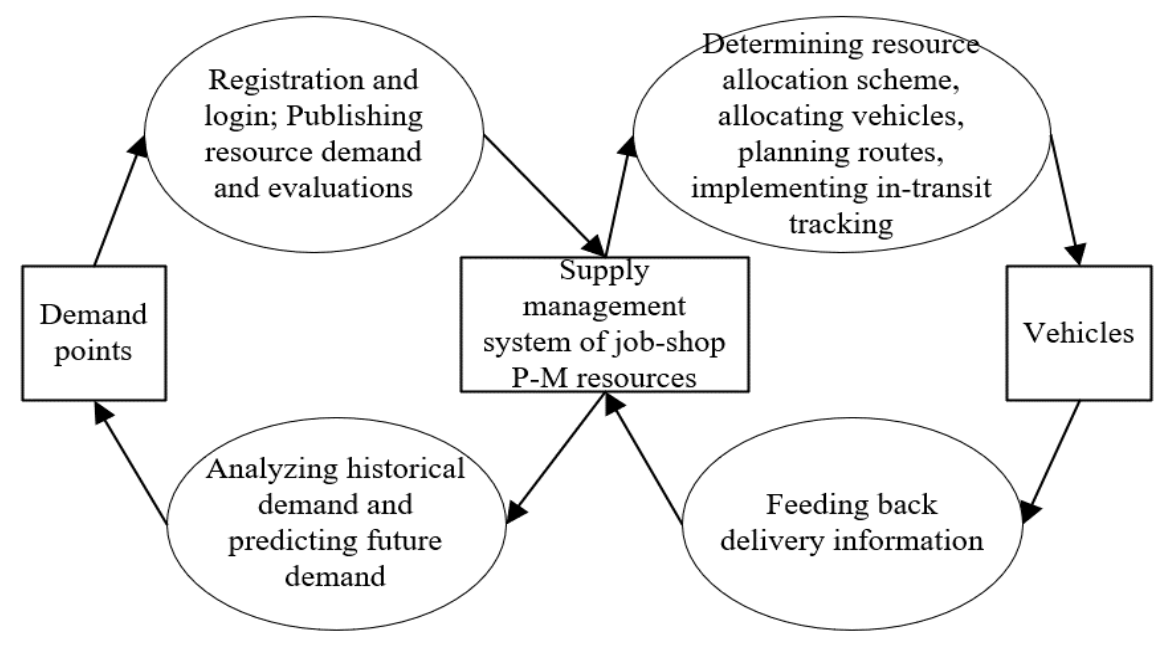

Figure 3: Operation mode of the system.

Based on the idea of aggregation and prediction, the P-M resource demand in the near future can be forecasted based on the historical information of job-shop P-M. The predicted demand can be added to the production control scheme to maximize the profit of job-shop P-M. Referring to the completion period, P-M resource demand may occur in any zone at any moment in future, in addition to the zones with actual demand. Let $\mu\left(\chi_{d i}\right)$ be the occurrence probability of P-M resource demand per unit time. If the prediction unit is small, then the probability $\mu\left(\chi_{d i}\right)$ is low. The deviation in resource demand prediction will drive up P-M cost or extend the completion period. To solve the problem, this paper combines each low probability RAZ with its adjacent prediction unit. Firstly, the occurrence probability $\mu\left(\chi_{d i}\right)$ of P-M resource demand in each prediction unit $d_{i}$ in the previous periods should be counted. If $\mu\left(\chi_{d i}\right)$ is relatively large, i.e., $\mu\left(\chi_{d i}\right)>C T^{\min -\mu}$, the demand will be directly predicted. If $\mu\left(\chi_{d i}\right)$ is relatively small, i.e., $\mu\left(\chi_{d i}\right)<C T^{m i n-\mu}$, the adjacent prediction units will be combined, until the occurrence probability of resource demand in the combined prediction unit $v$ is equal to or greater than $\mu\left(\chi_{d i}\right)$. However, there should not be too many combined RAZs. Otherwise, the delivery of P-M resources will 
become inefficient. The occurrence probability of P-M resource demand in a combined prediction unit can be calculated by:

$$
\mu\left(\chi_{v i}\right)=\sum_{i=1}^{m} \mu\left(\chi_{d i}\right)
$$

If $\mu\left(\chi_{d i}\right)>\mu\left(\chi_{v i}\right)$, the predicted P-M resource demand will be obtained.

\subsection{Allocation optimization modelling}

To reflect the P-M resource allocation situation in the supply management system of job-shop $\mathrm{P}-\mathrm{M}$ resources, the objective function of our $\mathrm{P}-\mathrm{M}$ resource allocation model was constructed in the light of three elements: transport distance cost, vehicle cost, and time window penalty cost. The travel distance of vehicles is positively correlated with the energy consumption of delivery. The transport distance cost $u_{1}$ refers to the energy consumed by a vehicle in executing the delivery task. Let $T_{X}=\{1,2, \ldots, m\}$ be the set of delivery stations to satisfy the actual demands, in which station $i$ corresponds to demand $i ; T_{d}=\{m+1, m+2, \ldots, m+n\}$ be the set of delivery stations to satisfy the predicted demands, in which station $i$ corresponds to demand $i ; T=\{1$, $2, \ldots, m+n\}$ be the set of delivery stations to satisfy all demands, including actual and predicted demands; $V=\left\{1^{\prime}, 2^{\prime}, \ldots,(m+n)^{\prime}\right\}$ be the set of demand points, in which demand $i$ corresponds to demand point $i^{\prime} ; L=\{1,2, \ldots, n\}$ be the set of vehicles; $M=\{T \cup V\}$ be the set of all delivery stations and demand points; $u$ be the travel cost per unit distance of each vehicle; $\xi_{i j} \geq 0$ be the distance between points $i$ and $j, i, j \in M$. Then, $u_{1}$ can be calculated by:

$$
u_{1}=\sum_{l \in L_{i}} \sum_{j \in M} u q_{i} \xi_{i j} a_{i j l}
$$

where, $a_{i j l}$ is the binary function reflecting whether vehicle $l$ moves from point $i$ to point $j$. If yes, $a_{i j l}=1$; otherwise, $a_{i j l}=0$.

$$
a_{i j l}=\left\{\begin{array}{l}
1, \text { vehicle } 1 \text { moves from point i to point } j \\
0, \text { otherwise }
\end{array} \forall l \in L, \forall i, j \in M\right.
$$

where, $q_{i}$ are the weights of actual and predicted demands:

$$
q_{i}=\left\{\begin{array}{l}
1, i \in(1,2, \ldots, m) \\
0.5, i \in(m+1, m+2, \ldots, m+n)
\end{array}\right.
$$

During P-M resource allocation, the number of vehicles is positively proportional to the fixed vehicle cost. This number can be characterized by the depreciation of vehicle in use. Let $u_{0}$ be the vehicle cost per unit resources; $q_{i}$ be the weights of actual and predicted demands; $n$ be the number of vehicles used in resource allocation. Then, the total fixed vehicle cost $u_{2}$ can be calculated by:

$$
u_{2}=\sum_{l \in L_{i}} \sum_{j \in M} u_{0} q_{i} b_{l}
$$

where, $b_{l}$ indicates whether the supply management system of job-shop P-M resources calls vehicle $l$ to deliver resources. If yes, $b_{l}=1$; otherwise, $b_{l}=0$.

$$
b_{l}=\left\{\begin{array}{l}
1, \text { the delivery center calls vehicle } l \\
0, \text { otherwise }
\end{array}\right.
$$

The supply management system of job-shop P-M resources has a high requirement on the timely completion of products. If a vehicle cannot complete the delivery in the required period, a penalty cost will be generated. Considering the completion time window of the products, any vehicle arriving before and after the time window for the P-M resource demand in any production phase will generate a penalty cost. Figs. 4 and 5 illustrate the time window of resource demand and penalty cost, respectively. 


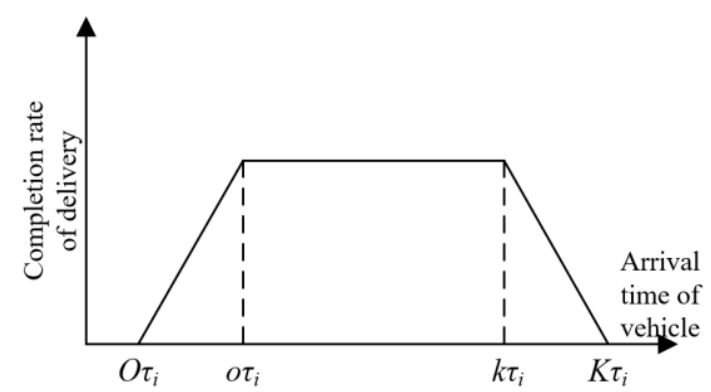

Figure 4: Time window of resource demand.

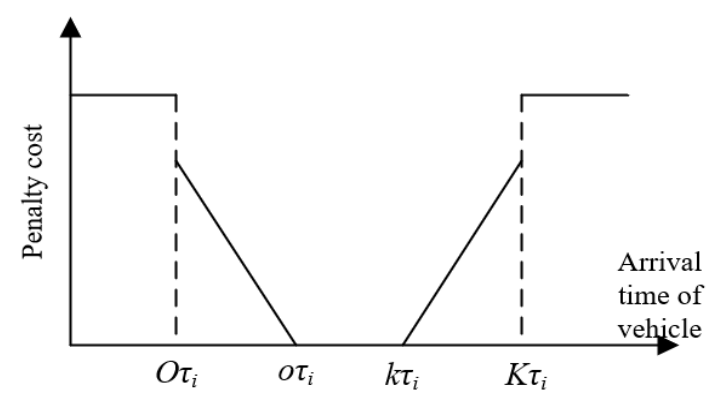

Figure 5: Penalty cost.

Let $\tau_{i j} \geq 0$ be the travel time between points $i$ and $j, j \in M$; $o \tau_{i}$ and $k \tau_{i}$ be the upper limit and lower limit of the time window for demand point $i$, i.e., the required period for the vehicle to arrive at the delivery station $i$, respectively; $\left[o \tau_{i}, k \tau_{i}\right], i \in M$ be the complete time window. After introducing a penalty coefficient $\beta$, the following penalty cost function can be established for the vehicles:

$$
u_{i}=\left\{\begin{array}{l}
\max \left\{\beta\left(o \tau_{i}-\tau_{i}\right), 0\right\} \\
\max \left\{\beta\left(\tau_{i}-k \tau_{i}\right), 0\right\} \\
0, o_{i} \leq \tau_{i} \leq b_{i}
\end{array}\right.
$$

The penalty cost $u_{3}$ can be expressed as:

$$
U_{3}=\sum_{i \in T} \max \left\{q_{i} \beta\left(o \tau_{i}-\tau_{i}\right), 0\right\}+\sum_{i \in T} \max \left\{q_{i} \beta\left(\tau_{i}-k \tau_{i}\right), 0\right\}
$$

Next is to model the vehicle allocation for resource delivery in the supply management system of job-shop P-M resources. The objective function of the model, which minimizes the total cost involving $u_{1}, u_{2}$, and $u_{3}$, can be established as:

$$
\begin{aligned}
& \min C=\sum_{l \in L_{i}} \sum_{j \in M} u q_{i} \xi_{i j} a_{i j l}+\sum_{l \in L_{i}} \sum_{j \in M} u_{0} q_{i} b_{l}+\sum_{i \in T} \max \left\{q_{i} \beta\left(o \tau_{i}-\tau_{i}\right), 0\right\} \\
& +\sum_{i \in T} \max \left\{q_{i} \beta\left(\tau_{i}-k \tau_{i}\right), 0\right\}
\end{aligned}
$$

During P-M resource allocation, the number of vehicles being allocated must be smaller than the total number of vehicles:

$$
\text { s.t. } \sum_{l \in L} \sum_{i, j \in M} a_{i j l} \leq L
$$

The P-M resource demand points are of equal importance in terms of accessibility, and each demand point can only be served by a vehicle at a time:

$$
\sum_{l \in L} \sum_{j \in V} a_{i j l}=1, \forall i \in T
$$

If a demand point is visited by a vehicle, then the corresponding delivery station must be visited by that vehicle: 


$$
\sum_{j \in V} a_{i j l}-\sum_{j \in V} a_{j i^{\prime} l}=0, l \in L, \forall i \in T
$$

The number of vehicles entering a delivery station must be balanced with that leaving that station:

$$
\sum_{i \in M} a_{i j l}-\sum_{j \in M} a_{j i^{\prime} l}=0, l \in L, \forall k \in M
$$

Let $W_{i j l}$ be the load of a vehicle moving from $i$ to $j$. Then, the load of vehicle $l$ before and after arriving at a delivery station should satisfy:

$$
\sum_{i \in M} \sum_{l \in L} W_{i t l}+\sum_{i \in M} \sum_{l \in L} a_{t j l} \xi_{t}=W_{t j l}, \forall j \in M
$$

The corresponding loading rate should satisfy:

$$
0 \leq W_{i j l} \leq W
$$

The start delivery time of a vehicle should satisfy:

$$
\tau_{S T-i} \geq 0, \forall i \in T
$$

\section{EXPERIMENTS AND RESULTS ANALYSIS}

The experiments use the dataset of the delivery trajectories for job-shop P-M resources of an automaker. Our model 1 was compared with moving average model 2, vector autoregression model 3, and linear regression model 4. The prediction performance of each model was evaluated by common metrics of demand prediction: root mean square error (RMSE) and mean absolute percentage error (MAPE). Our neural network was compiled in Python on Keras platform. The number of layers has a major impact on the capture of deep bidirectional spatiotemporal dependence. Hence, different layers of the network were tested. The test results (Table I) show that the prediction error initially decreased with the growing number of layers; once the number surpassed 6 , the prediction error began to fall. Thus, the model boasts the highest prediction accuracy, when the network has six layers.

Table I: Test results at different layers.

\begin{tabular}{|c|c|c|c|c|c|c|c|}
\hline \multicolumn{2}{|c|}{ Number of layers } & $\mathbf{3}$ & $\mathbf{4}$ & $\mathbf{5}$ & $\mathbf{6}$ & $\mathbf{7}$ & $\mathbf{8}$ \\
\hline \multirow{2}{*}{ Initial demand } & $R M S E$ & 24.26 & 23.42 & 23.27 & 23.19 & 23.81 & 24.22 \\
& $M A P E$ & $15.43 \%$ & $15.11 \%$ & $14.75 \%$ & $14.69 \%$ & $15.28 \%$ & $15.33 \%$ \\
\hline \multirow{2}{*}{ Final demand } & $R M S E$ & 18.71 & 18.62 & 18.53 & 18.45 & 18.65 & 18.82 \\
& $M A P E$ & $15.54 \%$ & $15.23 \%$ & $15.37 \%$ & $15.20 \%$ & $15.42 \%$ & $15.93 \%$ \\
\hline
\end{tabular}

The other model parameters were configured as follows: time step length, 6 ; length of longterm set of P-M resource demand, 8; length of short-term set of P-M resource demand, 4; coefficient of balance, 0.5 ; number of layers of local $\mathrm{CNN}$ and gated $\mathrm{CNN}, 3$; total number of fuzzy nodes, 128; learning rate, 0.01 .

Table II shows the test results of all models on the historical delivery dataset of job-shop P$\mathrm{M}$ resources in the same automaker. Model 2 had a large prediction error, as it only relies on historical demand for prediction. Models 3 and 4 outperformed Model 2, because they fully considered the linear spatial dependence of the prediction problem. Our model achieved the best prediction effect, for the comprehensive consideration of high-level time dependence. On the dataset of Job-shop 1, our model reduced the prediction error of the initial and final demands by $4.25 \% / 2.11 \%(R M S E)$ and $3.48 \% / 3.77 \%$ (MAPE). On the dataset of Job-shop 2, our model reduced the prediction error of the initial and final demands by $4.47 \% / 5.62 \%$ (RMSE) and $2.85 \% / 2.49 \%$ (MAPE). Fig. 6 compares the test results of different models, which more intuitively demonstrates the superiority of our model. 
Table II: Experimental results on different datasets.

\begin{tabular}{|c|c|c|c|c|c|}
\hline \multirow{2}{*}{\multicolumn{2}{|c|}{$\begin{array}{c}\text { Data source } \\
\text { Model }\end{array}$}} & \multicolumn{4}{|c|}{ Job-shop 1} \\
\hline & & 1 & 2 & 3 & 4 \\
\hline \multirow{2}{*}{ Initial demand } & $R M S E$ & 29.36 & 27.45 & 23.12 & 22.43 \\
\hline & $M A P E$ & $20.39 \%$ & $19.73 \%$ & $17.43 \%$ & $14.75 \%$ \\
\hline \multirow{2}{*}{ Final demand } & $R M S E$ & 23.86 & 23.58 & 19.46 & 19.03 \\
\hline & $M A P E$ & $19.58 \%$ & $19.26 \%$ & $17.42 \%$ & $16.23 \%$ \\
\hline \multicolumn{2}{|c|}{ Data source } & \multicolumn{4}{|c|}{ Job-shop 2} \\
\hline \multicolumn{2}{|c|}{ Model } & 1 & 2 & 3 & 4 \\
\hline \multirow{2}{*}{ Initial demand } & $R M S E$ & 12.21 & 11.46 & 9.86 & 7.45 \\
\hline & $M A P E$ & $25.72 \%$ & $25.82 \%$ & $23.12 \%$ & $22.58 \%$ \\
\hline \multirow{2}{*}{ Final demand } & $R M S E$ & 11.08 & 7.79 & 8.14 & 8.68 \\
\hline & $M A P E$ & $26.17 \%$ & $24.25 \%$ & $21.86 \%$ & $20.72 \%$ \\
\hline
\end{tabular}

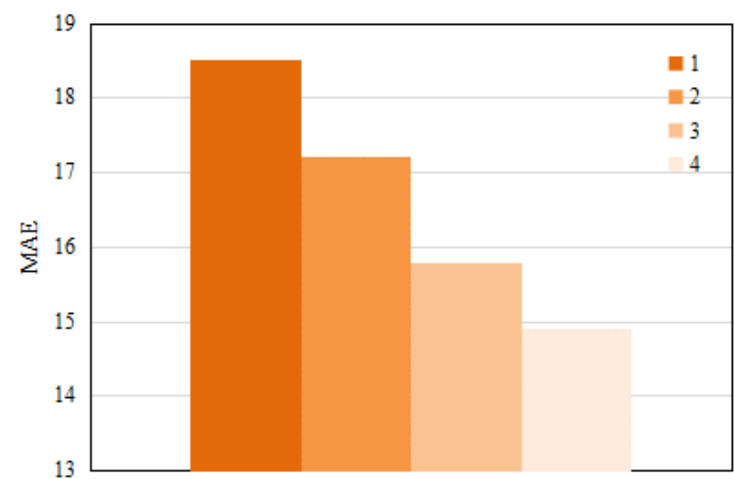

a)

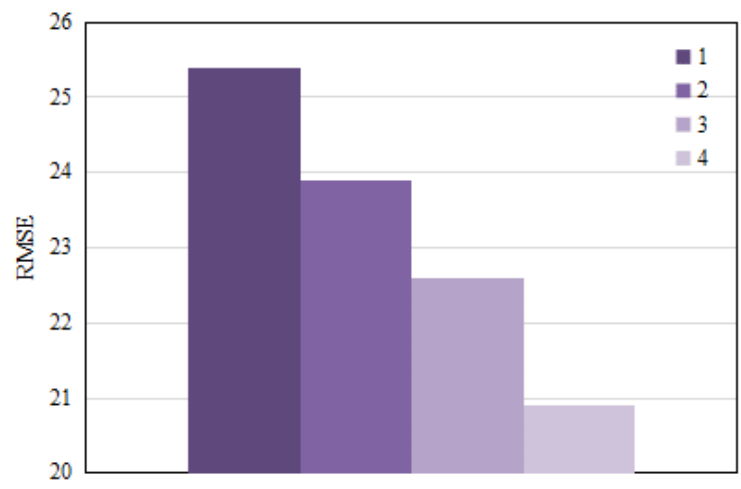

b)

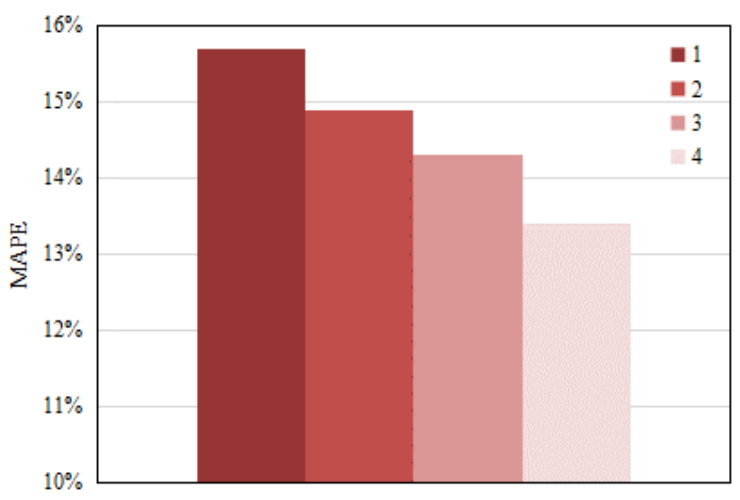

c)

Figure 6: Test results of 4 different models, regarding a) MAE, b) RMSE, c) MAPE.

Table III: Predicted demand.

\begin{tabular}{|c|c|c|c|c|c|}
\hline Serial number of predicted demand & $\mathbf{2 1}$ & $\mathbf{2 2}$ & $\mathbf{2 3}$ & $\mathbf{2 4}$ & $\mathbf{2 5}$ \\
\hline Pickup point & $(26,14)$ & $(23,10)$ & $(22,13)$ & $(24,15)$ & $(25,11)$ \\
Demand point & $(38,14)$ & $(14,26)$ & $(32,16)$ & $(22,11)$ & $(25,13)$ \\
Time window & $(60,121)$ & $(60,89)$ & $(61,122)$ & $(152,181)$ & $(360,386)$ \\
Demand & 3 & 1 & 2 & 3 & 5 \\
\hline Serial number of predicted demand & $\mathbf{2 6}$ & $\mathbf{2 7}$ & $\mathbf{2 8}$ & $\mathbf{2 9}$ & $\mathbf{3 0}$ \\
\hline Pickup point & $(25,13)$ & $(20,16)$ & $(25,12)$ & $(23,11)$ & $(22,18)$ \\
Demand point & $(26,12)$ & $(28,15)$ & $(22,11)$ & $(24,17)$ & $(21,10)$ \\
Time window & $(720,745)$ & $(1082,1102)$ & $(1254,1250)$ & $(1344,1545)$ & $(1788,1842)$ \\
Demand & 2 & 3 & 6 & 3 & 4 \\
\hline
\end{tabular}


The prediction unit was initialized as $3 \mathrm{~m} \times 3 \mathrm{~m} \times 10 \mathrm{~min}$ for P-M resource demand forecast. That is, each RAZ is a $9 \mathrm{~m}^{2}$ rectangle, and the delivery period lasts $10 \mathrm{~min}$. Each combined prediction unit should contain $2 \times 2$ prediction units at the most, that is, a combined prediction unit should consist of 2 RAZs and 2 periods at the most. Table III gives the qualified P-M resource demands predicted through simulation. Based on the historical data, a total of 10 demand points were predicted. Fig. 7 shows the distribution of all delivery nodes, including actual demand points, predicted demand points, and pickup points.

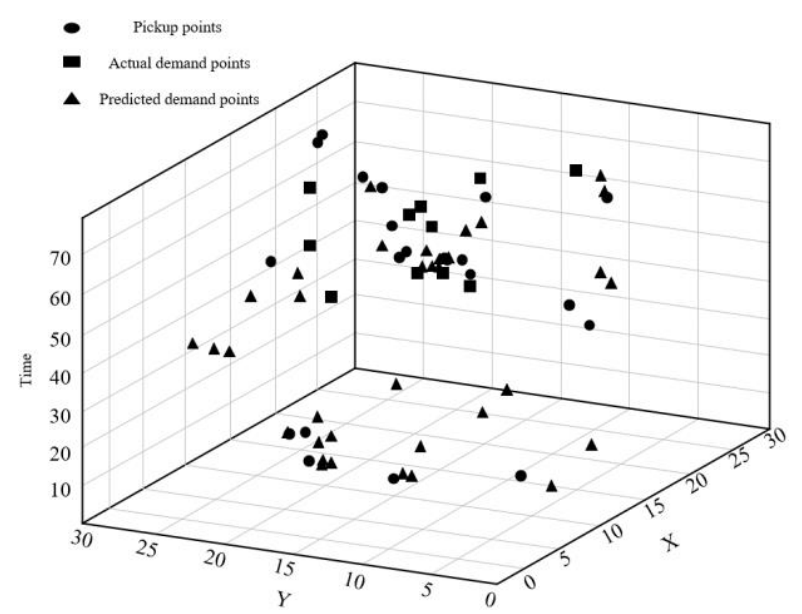

Figure 7: Distribution of P-M resource allocation nodes.

According to the survey data on the supply management system of job-shop P-M resources, the driving speed of vehicles was set to $10 \mathrm{~km} / \mathrm{h}$; the maximum number of available vehicles was set to 15 ; the unit travel cost, fixed cost, and penalty cost of vehicles were set to $0.5 \mathrm{yuan} / \mathrm{km}$, 10 yuan, and 1 yuan/min, respectively. The objective function of our allocation optimization model aims to minimize the total delivery cost. Multiple rounds of computing were carried out to optimize the value of the objective function, and to minimize the impact of random factors. Fig. 8 shows the vehicle trajectories of the optimal allocation scheme.

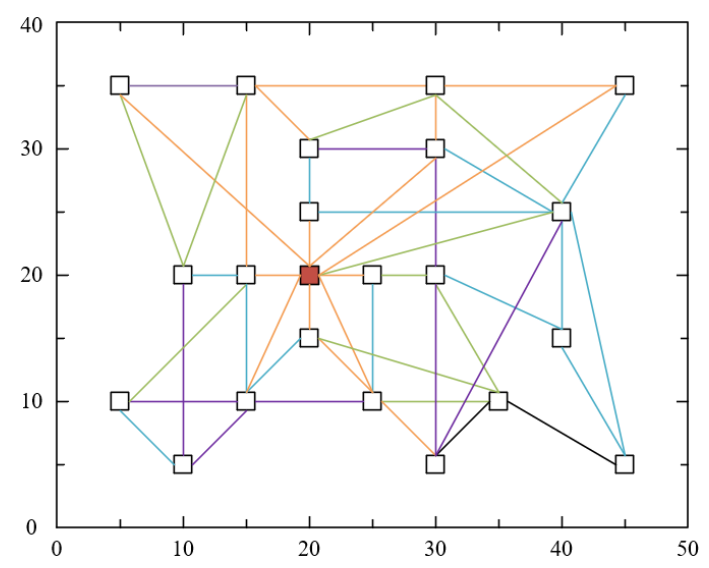

Figure 8: Vehicle trajectories of the optimal allocation scheme.

Then, the proposed dynamic P-M resource allocation strategy was compared with the random vehicle allocation strategy through experiments (Table IV). The comparison shows that our allocation scheme outshined the random allocation scheme in predicting the random P-M resource demand in the supply management system of job-shop P-M resources. Unlike our scheme, the random scheme does not consider the dynamic P-M resource demand, but only refers to the actual P-M resource demands to allocate vehicles. If any random demand arises, the random scheme needs to resort to vehicles that are not readily available. This will push up the fixed delivery cost, extend the delivery time, and affect the completion time. 
Table IV: Test results of different schemes.

\begin{tabular}{|c|c|c|c|c|c|c|c|}
\hline \multicolumn{2}{|c|}{ Serial number } & $\mathbf{1}$ & $\mathbf{2}$ & $\mathbf{3}$ & $\mathbf{4}$ & $\mathbf{5}$ & $\mathbf{6}$ \\
\hline Number of actual demands & 80 & 82 & 84 & 81 & 82 & 81 \\
\hline Number of virtual demands & 7 & 5 & 2 & 4 & 6 & 2 \\
\hline $\begin{array}{c}\text { Our } \\
\text { allocation } \\
\text { strategy }\end{array}$ & $\begin{array}{c}\text { Total number } \\
\text { of vehicles }\end{array}$ & 34 & 35 & 32 & 36 & 32 & 35 \\
\cline { 2 - 9 } $\begin{array}{c}\text { Rotal cost } \\
\text { allocation } \\
\text { strategy }\end{array}$ & $\begin{array}{c}\text { Total number } \\
\text { of vehicles }\end{array}$ & 32391.43 & 54685.56 & 60568.15 & 53491.34 & 52267.58 & 56179.49 \\
\cline { 2 - 9 } & Total cost & 56752.39 & 54031.65 & 61983.24 & 53582.58 & 51954.96 & 63512.73 \\
\hline
\end{tabular}

\section{CONCLUSIONS}

This paper investigates the demand forecast and allocation optimization of job-shop P-M resources, and manages to capture the static and dynamic spatial dependence of P-M resource volume. Based on demand prediction, the allocation optimization strategy was detailed for jobshop P-M resources, along with the objective function and constraints. The superiority of our model was demonstrated visually through a comparative experiment on different models. In addition, the authors presented the distribution of all distribution nodes, including actual demand points and pickup points, derived the optimal allocation scheme for P-M resources, and plotted the trajectories of vehicles under the optimal scheme. Finally, the proposed strategy was compared with the random vehicle allocation scheme. The comparison confirms the effectiveness of our dynamic P-M resource allocation strategy.

\section{ACKNOWLEDGEMENT}

This work was supported by Harbin University Youth doctoral research start fund project (No. HUDF2019207).

\section{REFERENCES}

[1] Wang, L. H. (2019). From intelligence science to intelligent manufacturing, Engineering, Vol. 5, No. 4, 615-618, doi:10.1016/j.eng.2019.04.011

[2] Gao, L.; Shen, W. M.; Li, X. Y. (2019). New trends in intelligent manufacturing, Engineering, Vol. 5, No. 4, 619-620, doi:10.1016/j.eng.2019.07.001

[3] Ojstersek, R.; Acko, B.; Buchmeister, B. (2020). Simulation study of a flexible manufacturing system regarding sustainability, International Journal of Simulation Modelling, Vol. 19, No. 1, 6576, doi:10.2507/IJSIMM19-1-502

[4] Wang, B.; Tao, F.; Fang, X.; Liu, C.; Liu, Y.; Freiheit, T. (2021). Smart manufacturing and intelligent manufacturing: a comparative review, Engineering, Vol. 7, No. 6, 738-757, doi:10.1016/j.eng.2020.07.017

[5] Sim, H. S. (2021). Hierarchical factor analysis methodology for intelligent manufacturing, Complexity, Vol. 2021, Paper 5593374, 13 pages, doi:10.1155/2021/5593374

[6] Pal, B. (2018). Optimal production model with quality sensitive market demand, partial backlogging and permissible delay in payment, RAIRO - Operations Research, Vol. 52, No. 2, 499-512, doi: $10.1051 / \mathrm{ro} / 2017068$

[7] Aoyama, T.; Nishi, T.; Zhang, G. (2017). Production planning problem with market impact under demand uncertainty, Journal of Advanced Mechanical Design, Systems, and Manufacturing, Vol. 11, No. 2, Paper 16-00532, 17 pages, doi:10.1299/jamdsm.2017jamdsm0019

[8] Onofrejova, D.; Janekova, J.; Grincova, A.; Soltysova, Z. (2020). Simulation and evaluation of production factors in manufacturing of fireplaces, International Journal of Simulation Modelling, Vol. 19, No. 1, 77-88, doi:10.2507/IJSIMM19-1-504 
[9] Kim, D.; Woo, J.; Shin, J.; Lee, J.; Kim, Y. (2019). Can search engine data improve accuracy of demand forecasting for new products? Evidence from automotive market, Industrial Management \& Data Systems, Vol. 119, No. 5, 1089-1103, doi:10.1108/IMDS-08-2018-0347

[10] Sverdrup, H. U.; Ragnarsdottir, K. V.; Koca, D. (2015). Aluminium for the future: modelling the global production, market supply, demand, price and long term development of the global reserves, Resources, Conservation and Recycling, Vol. 103, 139-154, doi:10.1016/j.resconrec.2015.06.008

[11] Jeong, S.; Na, W.; Kim, J.; Cho, S. (2018). Internet of Things for smart manufacturing system: trust issues in resource allocation, IEEE Internet of Things Journal, Vol. 5, No. 6, 4418-4427, doi:10.1109/JIOT.2018.2814063

[12] Tanaka, M.; Arima, S. (2018). Real-time allocation of multi-type production resource with due date grouping for MTO manufacturing, Proceedings of the 2018 International Symposium on Semiconductor Manufacturing, 4 pages, doi:10.1109/ISSM.2018.8651129

[13] Yin, L.; Luo, J.; Luo, H. (2018). Tasks scheduling and resource allocation in fog computing based on containers for smart manufacturing, IEEE Transactions on Industrial Informatics, Vol. 14, No. 10, 4712-4721, doi:10.1109/TII.2018.2851241

[14] Lin, T. Y.; Yang, C.; Zhuang, C.; Xiao, Y.; Tao, F.; Shi, G.; Geng, C. (2017). Multi-centric management and optimized allocation of manufacturing resource and capability in cloud manufacturing system, Proceedings of the Institution of Mechanical Engineers, Part B: Journal of Engineering Manufacture, Vol. 231, No. 12, 2159-2172, doi:10.1177/0954405415624364

[15] Ma, L.; Xu, F.; Wang, L.; Taslima, A. (2021). Impact of capital enrichment on resource allocation efficiency in China's manufacturing industry, Journal of Intelligent \& Fuzzy Systems, Vol. 41, No. 2, 4079-4095, doi:10.3233/JIFS-202856

[16] Zhu, D.; Xu, Z.; Xu, X.; Zhao, Q.; Qi, L.; Srivastava, G. (2021). Cognitive analytics of social media services for edge resource pre-allocation in industrial manufacturing, IEEE Transactions on Computational Social Systems, Vol. 8, No. 2, 500-511, doi:10.1109/TCSS.2021.3052231

[17] Delaram, J.; Houshamand, M.; Ashtiani, F.; Valilai, O. F. (2021). A utility-based matching mechanism for stable and optimal resource allocation in cloud manufacturing platforms using deferred acceptance algorithm, Journal of Manufacturing Systems, Vol. 60, 569-584, doi:10.1016/j.jmsy.2021.07.012

[18] Mashhadi, F.; Monroy, S. A. S. (2020). Deep learning for optimal resource allocation in IoTenabled additive manufacturing, Proceedings of the 2020 IEEE $6^{\text {th }}$ World Forum on Internet of Things, 6 pages, doi:10.1109/WF-IoT48130.2020.9221038

[19] Zhang, Y.; Yu, J. (2021). Research on high-efficiency resource allocation of multi-edge devices based on cloud manufacturing mode, Proceedings of the 2021 IEEE International Conference on Power, Intelligent Computing and Systems, 682-687, doi:10.1109/ICPICS52425.2021.9524141

[20] Zhou, B.; Bao, J.; Li, J.; Lu, Y.; Liu, T.; Zhang, Q. (2021). A novel knowledge graph-based optimization approach for resource allocation in discrete manufacturing workshops, Robotics and Computer-Integrated Manufacturing, Vol. 71, Paper 102160, 14 pages, doi:10.1016/ j.rcim.2021.102160

[21] Pei, Z.; Dai, X.; Yuan, Y.; Yi, W.; Chen, Y. (2021). Dynamic resource allocation of manufacturing network for emergency equipment, China Mechanical Engineering, Vol. 32, No. 7, 839-848, doi:10.3969/j.issn.1004-132X.2021.07.011

[22] Brintha, N. C.; Benedict, S.; Winowlin Jappes, J. T. (2020). Resource allocation in cloud manufacturing using bat algorithm, International Journal of Manufacturing Technology and Management, Vol. 34, No. 3, 296-310, doi:10.1504/IJMTM.2020.107309

[23] Yang, G. Z.; Chen, S. Q. (2020). Resource allocation algorithm and job scheduling of virtual manufacturing workshop, Academic Journal of Manufacturing Engineering, Vol. 18, No. 2, 155 161 